\title{
Serial ECG Analysis: Absolute Rather Than Signed Changes in the Spatial QRS-T Angle Should Be Used to Detect Emerging Cardiac Pathology
}

\author{
Agnese Sbrollini ${ }^{1}$, Marjolein C de Jongh ${ }^{2}$, C Cato ter Haar ${ }^{2}$, Roderick W Treskes ${ }^{2}$, Sumche Man ${ }^{2}$, \\ Laura Burattini ${ }^{1, *}$, Cees A Swenne ${ }^{2}$ \\ ${ }^{1}$ Università Politecnica delle Marche, Ancona, Italy \\ ${ }^{2}$ Leiden University Medical Center, Leiden, The Netherlands
}

\begin{abstract}
Background. Larger one-time values of spatial QRS-T angle (SA) are associated with risk. However, experience how serial changes in $S A(\triangle S A)$ should be interpreted is lacking. Even within normal limits, any $\triangle S A$ likely signifies electrical remodeling. This study aimed to assess the impact of choosing either $\triangle S A$ or $|\triangle S A|$ as one of a set of serial ECG difference features that constitute the input for our deep learning serial-ECG classifier (DLSEC).

Methods. DLSEC was trained and tested to detect emerging pathology in two serial ECG databases: a heart failure database and an acute ischemia database. Either $\triangle S A$ or $|\triangle S A|$ were among 13 features of serial-ECG differences. DLSEC was dynamically generated during learning, and testing area under the curve (AUC) of the receiver operating characteristic was computed.

Results. The DLSECs performed well in emerging heart failure as well as in acute ischemia: testing AUCs were $72 \%$ and $84 \%$ for the heart failure database and $77 \%$ and $83 \%$ for the ischemia database, for $\triangle S A$ or $|\triangle S A|$ among the features, respectively.

Conclusion. $|\triangle S A|$ among the features was superior to $\triangle S A$ in discriminating cases and controls. Our study supports the concept that any $\triangle S A$, irrespective of its sign, indicates a worsening clinical condition. Further corroboration requires studies in other clinical situations.
\end{abstract}

\section{Introduction}

Serial electrocardiography (ECG) is a promising clinical strategy to improve diagnosis and monitoring [1], [2]. It consists in the comparison of ECGs of the same patient, to reveal emerging pathology and to avoid confounding factors caused by inter-subject variability.

Several serial ECG features can reveal changes in clinical cardiac status, and one of them is the vectorcardiographic QRS-T spatial angle (SA), defined as the angle between the QRS-complex and the T-wave axes. $\mathrm{SA}$ is a measure of concordance/discordance between cardiac depolarization and repolarization [3] and has proven to be an important prognostic ECG index [4,5].
Until now, experience in how serial SA changes $(\Delta \mathrm{SA})$ should be interpreted is lacking: usually, clinicians interpret larger values of the SA as associated with risk, but possible pseudo-normalization can explain that in patients with acquired large SA values, new emerging pathology could decrease SA, while the clinical status deteriorates.

Thus, this study aims to assess the impact of choosing either $\triangle \mathrm{SA}$ or $|\Delta \mathrm{SA}|$ as one of a set of serial ECG features to diagnose emerging pathology, using a new deep learning serial-ECG classifier (DLSEC). To assess its general applicability, we evaluated the DLSEC algorithm in two different clinical situations.

\section{Material and Methods}

\subsection{Data}

Two databases with serial ECGs of control- and casepatients were considered. For each patient, the database contained two digital standard 10-second 12-lead ECGs, recorded at different time instants, and called the baseline ECG (BL-ECG) and the follow-up ECG (FU-ECG). In controls, the clinical status at the time at which BL-ECG and FU-ECG were made remained unchanged. In cases, the clinical status had changed in an unfavorable direction at the time at which the FU-ECG was made; hence, their FU-ECG has to be associated with newly arisen pathology.

The first database (heart failure database; 48/81 cases/controls)[4] was created to evaluate the ECG changes associated with emerging heart failure in subjects that had experienced a myocardial infarction in the past. The BL-ECGs of all patients were made in a clinically stable condition, at least 6 months after acute myocardial infarction. The FU-ECGs of controls were made about one year after the BL-ECGs, with unchanged clinical status. The FU-ECGs of cases was made when they presented themselves with evidence of emerging heart failure.

The second database (ischemia database; 84/398 cases/controls) [6] was created to evaluate ECG changes during acute ischemia. Control patients were retrospectively selected from the digital ECG database of the Leiden University Medical Centre; BL- and FU-ECGs were made during routine check-ups, about one year apart, 
ascertaining that the clinical status of these patients remained unchanged. The case patients belonged to the STAFF III database [7,8]. All cases had stable angina; acute ischemia was created by the balloon occlusions during elective percutaneous transluminal coronary angioplasty (PTCA). The BL-ECGs and the FU-ECGs were made before and after 3 minutes of balloon occlusion, respectively.

\subsection{Deep-Learning Serial ECG Classifier}

Our deep-learning serial ECG classifier (DLSEC) is a supervised multi-layer neural network (NN) [9]. To build and evaluate the classifier, we followed the classical scheme as represented in Figure 1, consisting of three main steps: 1) feature extraction and data division into a learning and a testing dataset, 2) construction and training of the classifier using the learning dataset, 3) performance evaluation using the testing dataset.

\subsubsection{Feature Extraction and Data Division}

All ECGs were analyzed by the Leiden ECG Analysis and Decomposition Software (LEADS) [10]. LEADS converts the 12-lead ECG into a vectorcardiogram (VCG) and measures a multitude of ECG and VCG variables in the averaged heartbeat. To represent the major cardiac electrical properties, we selected 13 ECG features. By subtracting the 13 BL-ECG features from the 13 FU-ECG features, we created for each patient a set of 13 ECG difference descriptors (DDs): the feature set. The 13 features are: QRS-duration difference (1), QT-interval difference (2), difference in maximal QRS-vector (3) and T-vector (4), QRS-integral vector (5) and T-integral vector (6) magnitude difference, QRS-complexity (7) and Twave complexity difference (8), magnitude of the ventricular-gradient difference vector (9), QRS-T spatialangle difference (10a) or magnitude of the QRS-T spatialangle difference (10b), heart-rate difference (11), magnitude of J-vector difference vector (12), T-wave symmetry difference (13).

Together, the 13 DDs characterize how the ECG of an individual has changed after the follow-up period. Newly emerging cardiac pathology will cause ECG changes, and, consequently, non-zero values of the 13 DDs. Due to these properties, the 13 DDs, as the input of the DLSEC, allow the DLSEC to discriminate between controls (in whom the ECG changes are thought to remain limited) and cases (in whom larger ECG changes are expected).

Each database is divided into learning and testing datasets with equal prevalences of cases and controls. The learning dataset is used to create and train the DLSEC ( $80 \%$ is used for training and $20 \%$ for validation), while the testing dataset was used to evaluate its performance.

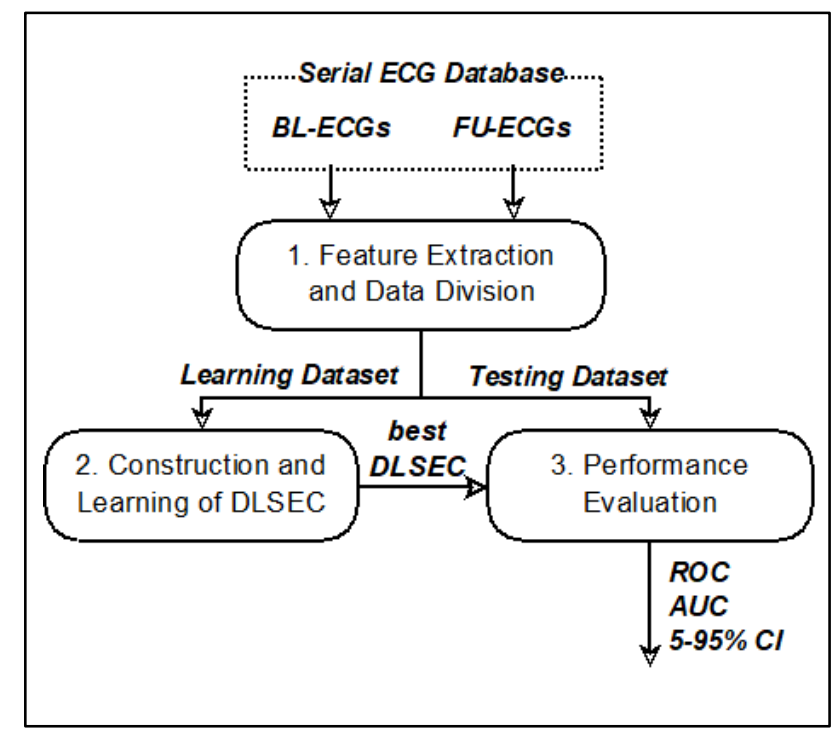

Figure 1. Main steps to construct the deep-learning serial ECG classifier and to evaluate its performance.

\subsubsection{Construction and Learning of the Deep-Learning Serial ECG Classifier}

Our deep-learning serial ECG classifier (DLSEC) is a supervised multi-layer neural network (NN) [9] with 13 inputs (the DDs) and one output, that assumes a value between 0 (definitely a control-patient) and 1 (definitely a case-patient). The DLSEC is dynamically formed during learning: its architecture and training of the neurons are alternated to reach the final DLSEC. Neurons (weights and biases: $[-1 ;+1]$; activation function: sigmoid) are trained with the scaled-conjugate-gradients algorithm [11].

The DLSEC construction procedure starts from a primitive NN, with one single neuron in one hidden layer: the first existing NN. From any given existing NN, new candidate structures are created. A candidate $\mathrm{NN}$ is a new $\mathrm{NN}$ that contains the neurons of the existing NN plus one additional neuron. Alternative candidate NNs differ as to the position of the new neuron in the structure: the new neuron can be located in one of the existing hidden layers, or it can constitute a new hidden layer. Limitations for alternative candidate structures are: the maximal number of hidden layers is 3 and, in case of multiple layers, the number of neurons in a given hidden layer cannot be larger than the number of neurons in the previous hidden layer.

In all valid alternative candidate NNs, the new neuron is initialized with random weight and bias, while the weights and biases of neurons that already existed are kept.

After initialization of the new neuron, the acceptability of this candidate $\mathrm{NN}$ must be assessed: a candidate $\mathrm{NN}$ is only acceptable if the new neuron helps the existing NN to reach a better training performance after one single initial learning iteration. If, after this single initial learning 
iteration, the training error is larger than the training error of the existing $\mathrm{NN}$, a next single initial learning iteration with a different initialization of the new neuron is attempted. This process is repeated till an initialization is found that renders the candidate acceptable, or till 500 attempts have been done without success: in that case the candidate is rejected. If all candidates are rejected, the existing NN is kept as the final DLSEC.

Acceptable candidate NNs enter the learning procedure, to investigate if they can outperform the existing NN. To this purpose, the final validation error of each candidate $\mathrm{NN}$ is compared with the validation error of the existing $\mathrm{NN}$ : if it is smaller, the candidate $\mathrm{NN}$ becomes the new existing NN. In case of multiple successful candidate NNs, the candidate $\mathrm{NN}$ with the lowest validation error is chosen to replace the existing $\mathrm{NN}$.

After an existing $\mathrm{NN}$ advancement, the structuring procedure starts anew. The procedure stops when 1) there are no acceptable candidate NNs, 2) no candidate $\mathrm{NN}$ is able to outperform the existing $\mathrm{NN}$, or 3) there are no misclassifications in the learning dataset.

Like in any optimization problem, there is never a guarantee that the optimal performance is reached. Thus, our algorithm constructs 100 DLSECs, each time starting from a different random initialization. For each realization, a receiver operating curve (ROC) [12] is constructed by varying the case-control decision threshold between 0 and 1 , thus computing the area under the curve (AUC). The resulting DLSEC is the one with the largest learning AUC.

\subsubsection{Performance Evaluation}

Finally, the testing dataset is used to evaluate the performance of the best DLSEC. This is done in a similar way as described above: a ROC is constructed by varying the case-control decision threshold between 0 and 1 , thus constructing the AUC and its $95 \% \mathrm{CI}$.

\subsection{QRS-T Spatial Angle Variants}

Purpose of the current study is to evaluate the impact of incorporating either the signed QRS-T spatial-angle difference, $\triangle \mathrm{SA}$ (difference descriptor 10a), or the absolute QRS-T spatial-angle difference, $|\Delta \mathrm{SA}|$ (difference descriptor 10b), in the feature vector. Consequently, two alternative DLSECs were constructed for each of the two serial ECG databases. The performances of these two variants were compared regarding their AUC and if they were statistically significantly $>0.5$ (DeLong's test [13]).

\section{Results}

Table 1 shows the structures and performances of the 4 DLSECs that were generated (two for the heart failure database and two for the ischemia database, each with either $|\Delta \mathrm{SA}|$ or $|\Delta \mathrm{SA}|$ as difference descriptor 10). The structure of the DLSECs differed strongly: the heart failure DLSECs required more neurons than the ischemia DLSECs, while the $|\triangle \mathrm{SA}|$ variant had less neurons than the $\triangle \mathrm{SA}$ variant for the heart failure database and more neurons for the ischemia database.

In both databases, the AUCs of the $\triangle \mathrm{SA}$ variants $(72 \%$ for the heart failure database and $77 \%$ for the ischemia database) were smaller than the AUCs of the $|\Delta \mathrm{SA}|$ variants $(84 \%$ for the heart failure database and $83 \%$ for the ischemia database). Moreover, the 5-95\% CI ranges for the $\triangle \mathrm{SA}$ variants $(26 \%$ for the heart failure database and $18 \%$ for the ischemia database) were larger than the 5-95\% CI ranges of the $|\Delta \mathrm{SA}|$ variants $(22 \%$ for the heart failure database and $16 \%$ for the ischemia database). Figure 2 shows the ROCs; all AUCs were statistically significantly larger than $0.5\left(\mathrm{P}\right.$ values $\left.<10^{-3}\right)$.

\section{Discussion}

The aim of this study was to evaluate the impact of choosing either $\Delta \mathrm{SA}$ or $|\Delta \mathrm{SA}|$ as one of a set of serial ECG features for the detection of emerging cardiac pathology. This feature set assessment was performed in two serial ECG databases: a database of patients with a healed myocardial infarction in which the case-patients had developed heart failure at follow-up, and a database of cardiac patients, in which the case-patients had acute ischemia as a consequence of balloon occlusions during PTCA. For each database, two DLSECs were developed, for either $\Delta \mathrm{SA}$ or $|\Delta \mathrm{SA}|$ in the feature set, respectively.

Table 1 shows that, although these differences did not reach statistical significance, the testing AUCs computed with the $|\Delta \mathrm{SA}|$ variants were larger than the testing AUCs computed with the $\triangle \mathrm{SA}$ variants in both databases. Additionally, the smaller 5-95\% confidence intervals

Table 1. Performance of the DLSECs computed with the two $\Delta \mathrm{SA}$ variants for the heart failure database and for the ischemia database. The structures, between brackets, denote the numbers of neurons in the first, second, and third hidden layers, respectively.

\begin{tabular}{|c|c|c|c|}
\cline { 3 - 4 } \multicolumn{2}{c|}{} & $\Delta \mathbf{S A}$ & $|\mathbf{\Delta S A}|$ \\
\hline \multirow{3}{*}{$\begin{array}{c}\text { Heart } \\
\text { Failure } \\
\text { Database }\end{array}$} & Structure & {$[241310]$} & {$\left[\begin{array}{lll}16 & 13 & 12\end{array}\right]$} \\
\cline { 2 - 4 } & $A U C(\%)$ & $72 *$ & $84 *$ \\
\cline { 2 - 4 } & $C I(\%)$ & {$[59 ; 85]$} & {$[73 ; 95]$} \\
\hline \multirow{3}{*}{$\begin{array}{l}\text { Ischemia } \\
\text { Database }\end{array}$} & Structure & {$[1200]$} & {$[1191]$} \\
\cline { 2 - 4 } & $A U C(\%)$ & $77 *$ & $83 *$ \\
\cline { 2 - 4 } & $C I(\%)$ & {$[68 ; 86]$} & {$[75 ; 91]$} \\
\hline
\end{tabular}

$: \mathrm{P}<10^{-3}$ when comparing the AUC with 0.5. 


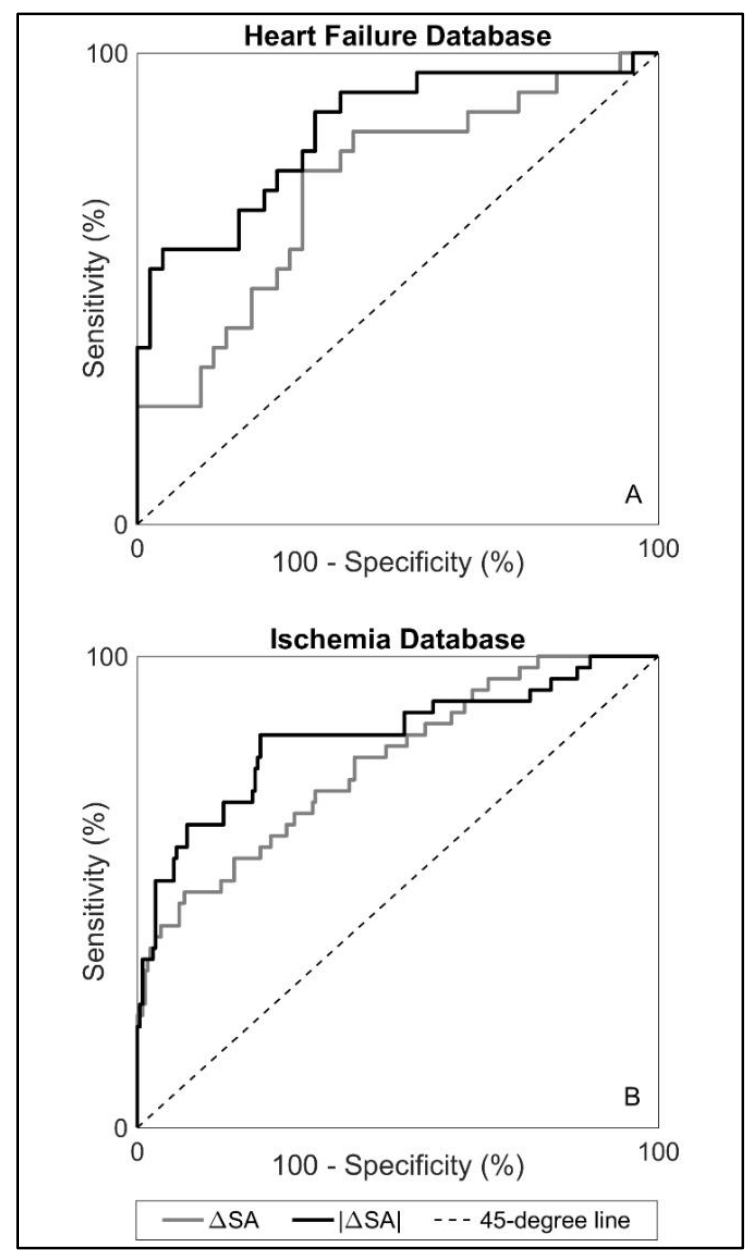

Figure 2. DLSEC ROCs computed for the heart failure (panel A) and ischemia (panel B) databases. Grey and black lines depict the $\Delta \mathrm{SA}$ and $|\Delta \mathrm{SA}|$ variants, respectively. The 45-degree lines represent $\mathrm{AUC}=0.5$.

confirm the superiority $|\Delta \mathrm{SA}|$ over $\Delta \mathrm{SA}$. Hence, the discriminatory power of the DLSEC in the detection of emerging pathology is stronger if the feature set considers changes in SA irrespective of the direction of change. This finding is likely to be explained by the possibility of pseudo-normalization: newly emerging pathology can mask existing pathology because both have an opposite effect on the ECG. Hence, a decrease of SA over time is not necessarily correlated with an improved clinical status.

\section{Conclusion}

Our study supports the concept that any change in SA, decrease or increase, can signal a worsening clinical status.

Future studies have to demonstrate if $|\Delta \mathrm{SA}|$ is also important in the detection of emerging cardiac pathologies other than heart failure or ischemia. A positive outcome of such studies would render $|\Delta \mathrm{SA}|$ an essential ECG variable in serial ECG analysis in standard clinical practice.

\section{References}

[1] J. Velez, W. J. Brady, A. D. Perron, and L. Garvey, "Serial electrocardiography," Am. J. Emerg. Med., vol. 20, no. 1, pp. 43-49, Jan. 2002.

[2] C. A. Swenne, "Improved STEMI diagnosis by serial ECG analysis," J. Electrocardiol., vol. 48, no. 1, pp. 99-100, Jan.Feb. 2015.

[3] S. Man, A. C. Maan, M. J. Schalij, and C. A. Swenne, "Vectorcardiographic diagnostic \& prognostic information derived from the 12-lead electrocardiogram: Historical review and clinical perspective," J. Electrocardiol., vol. 48, no. 4, pp. 463-475, Jul.-Aug. 2015.

[4] M. C. De Jongh et al., "Progression towards heart failure after myocardial infarction is accompanied by a change in the spatial QRS-T angle," Comput. Cardiol., vol. 44, pp. 25 Sep. 2017.

[5] C. J. W. Borleffs et al., "Predicting ventricular arrhythmias in patients with ischemic heart disease: clinical application of the ECG-derived QRS-T angle," Circ. Arrhythm. Electrophysiol., vol. 2, no. 5, pp. 548-554,Oct. 2009.

[6] R. W. Treskes et al., "Performance of ST and ventricular gradient difference vectors in electrocardiographic detection of acute myocardial ischemia," J. Electrocardiol., vol. 48, no. 4, pp. 498-504, Jul.-Aug. 2015.

[7] S. G. Warren and G. S. Wagner, "The STAFF studies of the first 5 minutes of percutaneous coronary angioplasty balloon occlusion in man," J. Electrocardiol., vol. 47, no. 4, pp. 402 407, Jul.-Aug. 2014.

[8] A. L. Goldberger et al., "PhysioBank, PhysioToolkit, and PhysioNet: components of a new research resource for complex physiologic signals," Circulation, vol. 101, no. 23, pp. e215-e220, Jun. 2000.

[9] A. Goodfellow, I. Bengio, and Y. Courville, "Deep learning," MIT Press, 2016.

[10] H. H. M. Draisma et al., "LEADS: An interactive research oriented ECG/VCG analysis system," Comput. Cardiol., vol. 32, pp. 515-518, Sep. 2005.

[11] M. F. Møller, "A scaled conjugate gradient algorithm for fast supervised learning," Neural Networks, vol. 6, no. 4, pp. 525-533, 1993.

[12] C. E. Metz, "Basic principles of ROC analysis," Semin. Nucl. Med., vol. 8, no. 4, pp. 283-298, Oct. 1978.

[13] E. R. DeLong, D. M. DeLong, and D. L. Clarke-Pearson, "Comparing the areas under two or more correlated receiver operating characteristic curves: a nonparametric approach," Biometrics, vol. 44, no. 3, p. 837, 1988.

Address for correspondence.

Laura Burattini, $\mathrm{PhD}$

Università Politecnica delle Marche,

Department of Information Engineering,

Via Brecce Bianche,

60131 Ancona, Italy.

E-mail address.1.burattini@univpm.it. 